\title{
Caracterización nutricional de niños con errores innatos del metabolismo de proteínas, en una consulta privada de nutrición en Bogotá, Colombia
}

\author{
Nutritional status of children with inborn \\ errors of protein metabolism in a private nutritional \\ practice in Bogotá, Colombia
}

\begin{abstract}
Little is known about the nutritional consequences of inborn errors of metabolism (IEM) without neonatal diagnosis in Colombia. The aim of our study was to describe the nutritional characteristics of individuals with IEM who attended a nutritional private practice in Bogotá, Colombia, and to evaluate whether this status is consequence of the disease or other social or demographic variables. This was a descriptive observational study with a cross-sectional design, in which anthropometrical measurements were taken along with a diet analysis and the recollection of social and demographic variables. A sample of 22 individuals was gathered: Forty five \% women, $36 \%$ infants, $59 \%$ resided in an urban area and $77 \%$ had a low socioeconomic status (SES). More than $40 \%$ had an adequate nutritional status and 45\% suffered growth retardation; no differences were found according to gender, area of residence, SES nor age group. Nearly $90 \%$ had adequate treatment adherence, fulfilling their nutritional requirements according to age, disease and limiting amino acids. The only difference found in dietary intake was among age groups, in which the intake per kg of weight decrease as the individual got older. We concluded that the main nutritional outcome in patients with IEM was growth retardation and it can be attributed to the disease rather than other social or demographic variables.

Key words: inborn errors of metabolism, growth retardation, dietary intake, nutritional management, anthropometric assessment.
\end{abstract}

\section{INTRODUCCIÓN}

Los errores innatos del metabolismo (EIM) son un grupo de enfermedades monogénicas de herencia autosómica recesiva, causadas por una mutación genética que tiene como efecto la producción de una proteína anómala, y que conlleva a la alteración del funcionamiento fisiológico de la célula. Las manifestaciones clínicas de estas proteínas alteradas van a ser muy variadas, y aparecen fundamentalmente en las etapas precoces de la vida. A pesar de que cada uno de los EIM tiene una incidencia muy baja, la incidencia acumulada para el conjunto de todos ellos es de 1 por cada 500 recién nacidos vivos, considerando que existen aproximadamente más de 700 tipos diferentes (1).

La mayoría de los EIM se manifiestan en la edad pediátrica, desde las primeras horas de vida y hasta la adolescencia,
Liliana Ladino M. (1)

Erika Ochoa O. (2)

(1) Grupo de Investigación Alimentos, Nutrición y Salud, Pontificia Universidad Javeriana, Bogotá D.C., Colombia. (2) Departamento de Nutrición y Bienestar Integral, Instituto Tecnológico y de Estudios Superiores de Monterrey, Campus Ciudad de México, México.

Dirigir la correspondencia a: Profesora

Liliana Ladino Meléndez Departamento de Nutrición y Bioquímica Facultad de Ciencias Pontificia Universidad Javeriana Cra. 7 No 43-82 Ed. Carlos Ortíz, Oficina 613 Bogotá, Colombia Fono: (57-1)3208320 Ext 4063 Email: adino./@javeriana.edu.cd

Este trabajo fue recibido el 19 de Junio de 2012 y aceptado para ser publicado el 28 de Octubre de 2012.

con síntomas y signos similares a otras patologías, por lo que fácilmente pueden confundirse con otras enfermedades menos frecuentes. El diagnóstico de los EIM es complejo y generalmente tardío, sobretodo en países como Colombia, donde no se cuenta con un programa de tamizaje neonatal para EIM, por lo que la mayoría de los niños en quienes se tiene la sospecha de un EIM son diagnosticados con el apoyo de pruebas bioquímicas y/o moleculares que son enviadas a países que disponen del equipo para realizar el diagnóstico; como Chile, Costa Rica, Brasil, Cuba, en Latinoamerica. Al no diagnosticar a tiempo la enfermedad, se presentan secuelas importantes, en el neurodesarrollo y ocasionando retardo mental como en el caso de los pacientes con fenilcetonuria, y en algunos otros como las acidemias orgánicas, el estado nutricional es el más afectado. La prevención de estas secuelas 
con un diagnóstico oportuno es el enorme desafío al que se enfrentan los pediatras; por ello, es necesario que los clínicos estén familiarizados con los síntomas y signos más comunes en los EIM (2).

Establecido el diagnóstico se inicia la terapia correspondiente a través de un seguimiento sistemático y estricto. Diversos protocolos sugieren que la evaluación nutricional se realice al momento del diagnóstico, a los 15 días, y posteriormente una vez al mes, la que permite realizar toda las adecuaciones de nutrientes con respecto a patrones estándares, prescribir las fórmulas lácteas especiales para cada una de las patologías (3), evaluar y ajustar de acuerdo a valores sanguíneos, el sustrato acumulado y detectar deficiencias o excesos de nutrientes; evaluar el estado nutricional y los requerimientos de nutrientes según parámetros antropométricos, edad y estado de salud. Todo esto enmarcado con la educación nutricional a los padres y al niño es esencial para lograr una buena adherencia y éxito del tratamiento nutricional.

Otra de las evaluaciones que debe realizarse de forma estricta, es la evaluación bioquímica, que se debe hacer con una frecuencia mínima de una vez al mes. Se determina el nivel de los aminoácidos y/o sus metabolitos, por análisis de aminoácidos, ácidos orgánicos, acilcarnitinas. Debido a las restricciones de alimentos naturales, es necesario realizar exámenes de rutina como el cuadro hemático, proteínas séricas, y algunos minerales o elementos trazas, una o dos veces al año.

Para el seguimiento nutricional existen múltiples protocolos internacionales $(1,2,9,4)$ todos basados en la experiencia de cada grupo de trabajo. Es importante señalar que el adecuado manejo nutricional controla el desbalance metabólico, logra el control metabólico global del paciente y previene las consecuencias desfavorables de la enfermedad, pudiendo mejorar la calidad de vida del niño y su familia. En nuestro medio, no se conocía hasta el momento, un estudio que describa las características nutricionales de los niños que han sido diagnosticados con algún tipo de EIM.

El objetivo de este estudio fue describir las principales características nutricionales de niños con EIM de proteínas, que asisten a una consulta privada de nutrición en Bogotá, Colombia. De forma secundaria, se describen las diferencias del estado nutricional e ingesta dietética de acuerdo a sexo, nivel socioeconómico, tipo de vivienda, grupo etáreo y patología.

\section{SUJETOS Y MÉTODOS}

Estudio descriptivo transversal observacional de pacientes con diagnóstico de algún tipo de EIM de proteínas, diagnosticados en una consulta médica especializada (neuropediatra, genetista y bioquímico), que asisten a una única consulta privada de nutrición, remitidos de diversos centros por el médico tratante, en Bogotá, Colombia, entre el 30 de enero de 2010 hasta el 9 de marzo del 2012. Los datos fueron tomados de la consulta más reciente.

Datos demográficos: Género (femenino, masculino); grupo etáreo (lactante: menores de 2 años, preescolar: entre 2 y 6 años, escolar: entre 7 y 12 años, adolescente: mujeres entre 10 y 17 años y hombres entre 13 y 17 años, adulto: mayor a 18 años); lugar de vivienda (urbano o rural en Bogotá o fuera de Bogotá); nivel socioeconómico familiar determinado por el estrato reportado en los recibos de servicios públicos de las viviendas, (según estrato socioeconómico bajo: 1 y 2, medio: 3 y 4, alto: 5 y 6) y diagnóstico de EIM de proteínas.

Antropometría: Se tomó la fecha de nacimiento para estimar la edad exacta en años y meses (se realizó aproximación al siguiente mes si se tenia más de 21 días cumplidos).
Se realizaron mediciones de peso $(P)$ en una báscula infantil SECA 374 con precisión de $10 \mathrm{~g}$ para menores de 2 años y báscula de resortes SECA con precisión de $100 \mathrm{~g}$ para mayores de 2 años, longitud (L) con infantómetro portátil SECA 416 con precisión de $0.5 \mathrm{~cm}$ en menores de 2 años, talla $(T)$ para mayores de 2 años con estadímetro portátil SECA 213 con precisión de $0.1 \mathrm{~cm}$, perímetro cefálico $(P C)$ para menores de dos años con cinta de fibra de vidrio de $150 \mathrm{~cm}$ y precisión de $+/-1 \mathrm{~mm}$, perímetro del brazo (PB) con cinta de fibra de vidrio de $150 \mathrm{~cm}$ y precisión de $1 \mathrm{~mm}$, y pliegue cutáneo del tríceps (PCT) con plicómetro Slim Guide con precisión de 1 $\mathrm{mm}$. Con las mediciones mencionadas se calculó el puntaje de los indicadores antropométricos: peso para la edad (P/E) de Gómez (5), peso para la longitud-talla (P/L-T) y longitudtalla para la edad (L-T/E) de Waterloow (6), índice de masa corporal para la edad (IMC/E), perímetro del brazo para la edad (PB/E) y pliegue cutáneo del tríceps para la edad (PCT/E), según los patrones de crecimiento y puntos de corte de la Resolución 2121 para Colombia, y la Organización Mundial de la Salud (OMS) (7) para todos los niños y niñas de 0-18 años. En pacientes mayores de 18 años se interpretó el IMC según el Consenso de la Sociedad Española para el Estudio de la Obesidad (SEEDO) (8). Los porcentajes de reservas corporales de masa magra (RMM) y grasa (RMG) se evaluaron según los puntos de corte de Frisancho (9) para RMM y RMG.

Dietética: Se tomaron datos sobre: la prescripción dietaría, aporte de energía expresado en Kilocalorías día (Kcal/ día) y Kilocalorías por Kilogramo día (Kcal/Kg/día), valor energético total (VET) expresado en porcentaje (\%), aporte de macronutrientes expresados en gramos por Kilogramo día (g/ $\mathrm{Kg} /$ día), aporte de proteínas dadas por la fórmula especial libre de aminoácidos correspondientes según EIM y fórmula láctea infantil si corresponde, expresado en \%, aporte de aminoácidos dependiendo del EIM expresado en miligramos por Kilogramo día (mg/Kg/día), miligramos día (mg/día) o gramos día (g/día).

Análisis estadístico: Se tabularon todos los datos recolectados en una base de datos en Excelmac 2011 y se analizó con el programa estadístico STATA 2006. Se realizó una estadística descriptiva, expresándose en media \pm desviación estándar, rangos mínimo y máximo y proporciones. Se utilizó la prueba de Chi2 para el análisis entre variables categóricas (sexo, tipo de vivienda, grupo etáreo, nivel socioeconómico y estado nutricional). Se analizó la normalidad de variables continuas por la prueba estadística de Shapiro-Wilk. Las variables que no presentaron distribución normal (aporte energético y proteico) se expresaron como medianas (rango intercuartil) y se analizaron con estadística no paramétrica. Para las diferencias entre sexo, vivienda y nivel socioeconómico se aplicó la prueba de MannWhitney; las diferencias entre grupos etáreos se analizaron por la prueba de Kruskal-Wallis.

Comité de Ética: Este estudio fue aprobado por el Comité de Ética de la Facultad de Ciencias de la Pontificia Universidad Javeriana. No se requirió de consentimiento informado escrito, ya que según el artículo 11 de la resolución 8430 de 1993 del Ministerio de Salud de Colombia, esta investigación se clasifica sin riesgo por ser una investigación documental retrospectiva con revisión de historias clínicas y para garantizar la anonimización de la información, únicamente el investigador principal conocía los nombres de cada una de las historias clínicas.

\section{RESULTADOS}

Se realizó una caracterización nutricional a los 22 pacientes con EIM de proteínas (20 con diagnóstico tardío y 2 con 
diagnóstico neonatal) que asisten a una consulta privada de nutrición en Bogotá, Colombia. La muestra estudiada tenía edad promedio de $8.4 \pm 1.8$ años (rango de 6 meses hasta 26 años), de los cuales (36.4\%) eran lactantes y (31.8\%) prescolares y escolares y (31.8\%) escolares; en total 12 de 22 (54.5\%) eran hombres. La mayoría residía en zonas urbanas (13 de 22), de los 9 individuos que residía en zona rural, 7 eran residentes en Bogotá. En cuanto a nivel socioeconómico, prevalece el estrato bajo en 17 (77.3\%) de los individuos. (tabla 1).

Para futuros análisis se decidió agrupar y comparar a los individuos por tipo de vivienda en: urbana contra rural (sin diferenciar si es en Bogotá o no) así como por NSE bajo contra

\section{TABLA 1}

Variables descriptivas

\begin{tabular}{|c|c|c|}
\hline Variables & $\begin{array}{c}n \\
(\mathrm{~N}=22)\end{array}$ & $\%$ \\
\hline Sexo (M/F) & $12 / 10$ & $54.5 / 45.5$ \\
\hline \multicolumn{3}{|l|}{ Grupos etáreos } \\
\hline Lactantes & 8 & 36.4 \\
\hline Preescolares y escolares & 7 & 31.8 \\
\hline Adolescentes y adultos & 7 & 31.8 \\
\hline \multicolumn{3}{|l|}{ Tipo de vivienda } \\
\hline Urbana & 8 & 36.4 \\
\hline Urbana Bogotá & 5 & 22.7 \\
\hline Rural & 2 & 9.1 \\
\hline Rural Bogotá & 7 & 31.8 \\
\hline \multicolumn{3}{|l|}{ Nivel Socioeconómico (NSE) } \\
\hline Bajo & 17 & 77.3 \\
\hline Medio & 2 & 9.1 \\
\hline Alto & 3 & 13.6 \\
\hline
\end{tabular}

\section{GRÁFICA 1}

Diagnóstico de errores innatos del metabolismo.
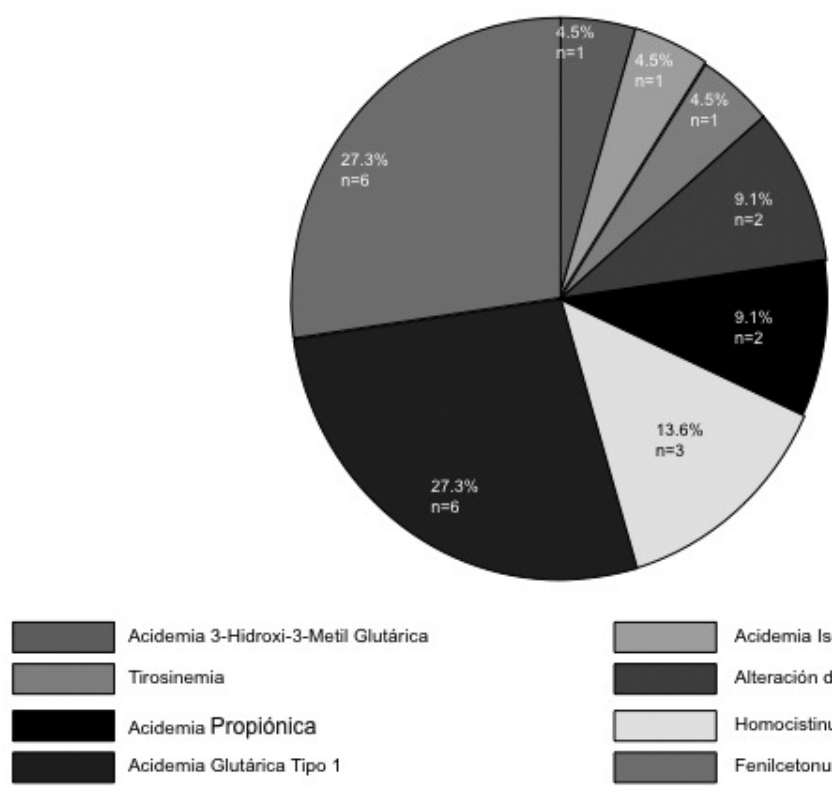

Acidemia 3-Hidroxi-3-Metil Glutárica

Tirosinemia

Acidemia Propiónica

Acidemia Glutárica Tipo 1

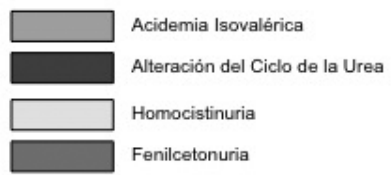


medio y alto. Dicha decisión se tomó en base al pequeño número muestral por categoría que impedía el análisis estadístico.

En nuestra limitada muestra la fenilcetonuria (PKU) y la acidemia glutárica tipo 1 (AG1) fueron las más prevalentes, representando más del $50 \%$ de los pacientes estudiados. La homocistinuria (HCU) se presentó en tres de los 22 individuos, acidemia propiónica (AP) en dos, alteración del ciclo de la urea $(A C U)$ en dos mientras que la tirosinemia tipo 1, academia isovalérica (AIV) y la acidemia 3-hidroxi-3-metil glutárica, en conjunto, fueron la menos prevalentes $(n=3,13.6 \%)$. (gráfico 1)

La valoración antropométrica permitió obtener el peso en todos los individuos de la muestra sin embargo sólo se pudo obtener la talla en 19 individuos, impidiendo evaluar todos los indicadores. Se calcularon los indicadores peso para la edad $(\mathrm{P} / \mathrm{E})$ en los 15 individuos menores a 10 años; talla o longitud para la edad (T/E) en 15 individuos ya en tres niños (dos lactantes y un preescolar) la longitud/talla no pudo ser evaluada y a que el indicador no es válido para adultos $(n=4)$; peso para la talla $(\mathrm{P} / \mathrm{T})$ se obtuvo en todos aquellos con medición de talla menor a 1.20m $(n=12)$ y, finalmente, el índice de masa corporal (IMC) en 19 individuos, de los cuáles se evaluó el indicador IMC para la edad (IMC/E) en 15 pacientes de edad pediátrica e IMC con puntos de corte según SEEDO en los cuatro adultos. Las reservas corporales no son presentadas ya que existió gran variabilidad y probabilidad de error.

En la tabla 2 se muestran los indicadores antropométricos previamente descritos. En la evaluación de los indicadores para edad pediátrica claramente se observa una gran dispersión de los datos (confirmando la necesidad de realizar estadística no paramétrica al comparar entre sexo, grupos etáreos, zona de vivienda y nivel socioeconómico).

Sin embargo, se pudo determinar que es una población que en su mayoría tenían riesgo de talla baja ya que al analizar individualmente los casos (gráfico 2) encontramos que sólo 4 individuos (27\%) presentaban talla adecuada para la edad mientras que 3 de ellos tenían riesgo de talla baja (puntaje z

TABLA 2

Indicadores antropométricos

\begin{tabular}{cccc}
\hline $\begin{array}{c}\text { Peso/Edad } \\
\text { z-score } \\
(n=15)\end{array}$ & $\begin{array}{c}\text { Peso/Talla } \\
\text { z-score } \\
(n=12)\end{array}$ & $\begin{array}{c}\text { Talla/Edad } \\
\text {-score } \\
(n=15)\end{array}$ & $\begin{array}{c}\text { IMC/Edad } \\
z \text {-score } \\
(n=15)\end{array}$ \\
$-1.5 \pm 1.3$ & $-0.6 \pm 1.6$ & $-1.9 \pm 1.6$ & $-0.4 \pm 1.4 \quad 23.4 \pm 1.4$ \\
\hline
\end{tabular}

Valores expresados en media \pm desviación estándar

Evaluación de talla y peso.

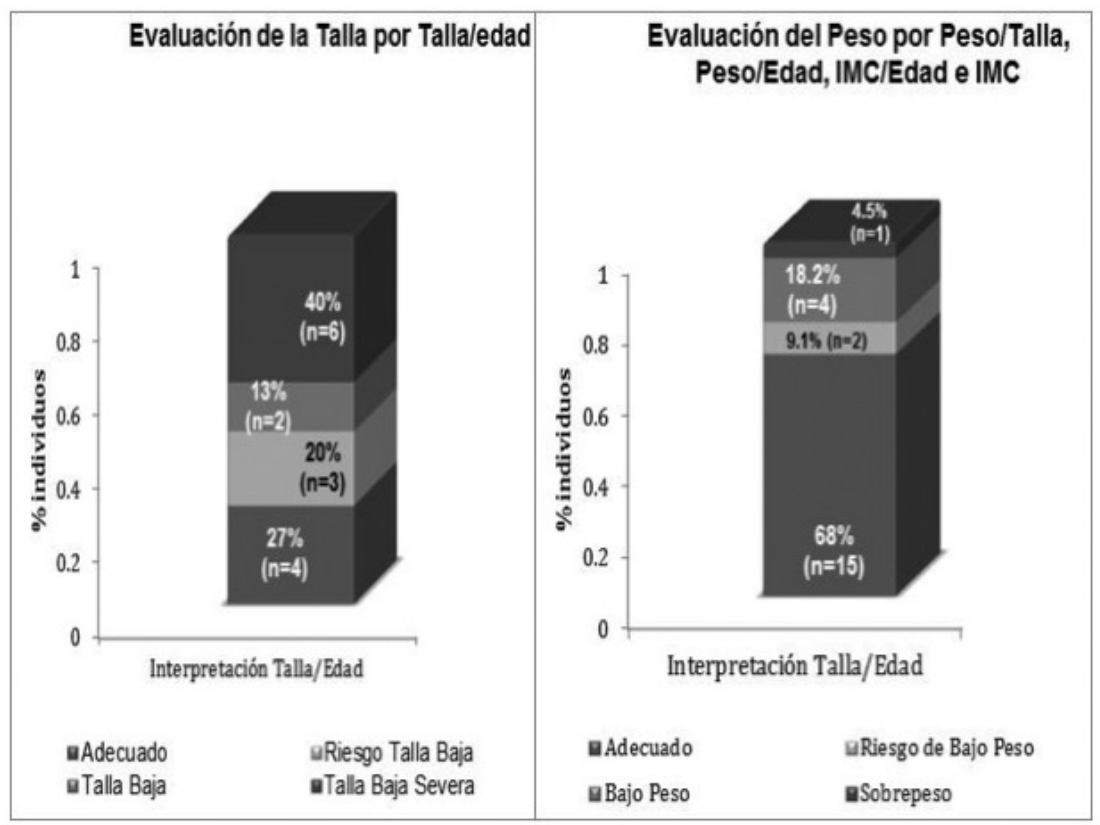


de -1 a -2 ) y los 8 individuos restantes (53\%) cursan con talla baja (puntaje z menor a -2).

En cuanto al peso encontramos que la mayoría $(n=15)$ presentaba normopeso mientras que $6(27.3 \%)$ cursaban con riesgo de bajo peso o bajo peso (encontrándose un caso con bajo peso severo tipo Kwashiorkor). Es importante mencionar que en la muestra también encontramos un caso atípico con sobrepeso.

\section{GRÁFICA 3}

Diagnóstico antropométrico.

- Eutrófico

= Desnutrición aguda

- Desnutrición crónica

- Desnutrición crónica agudizada

- Sobrepeso

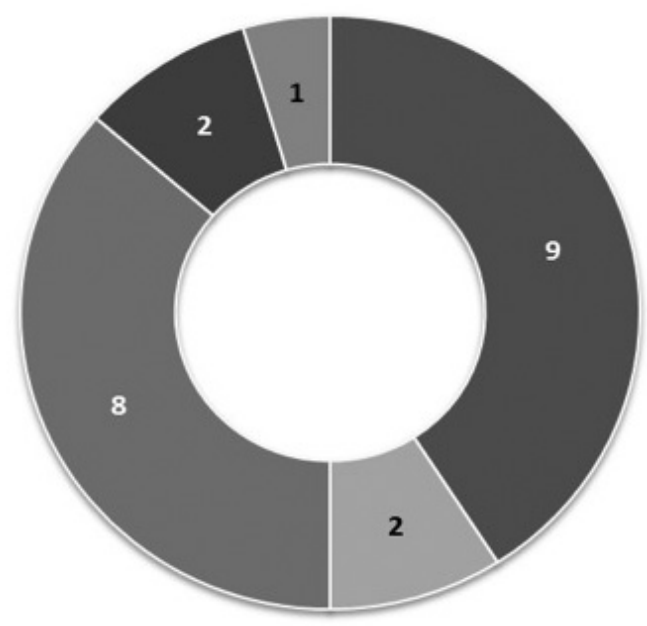

GRÁFICA 4

Diagnóstico nutricional por patología.

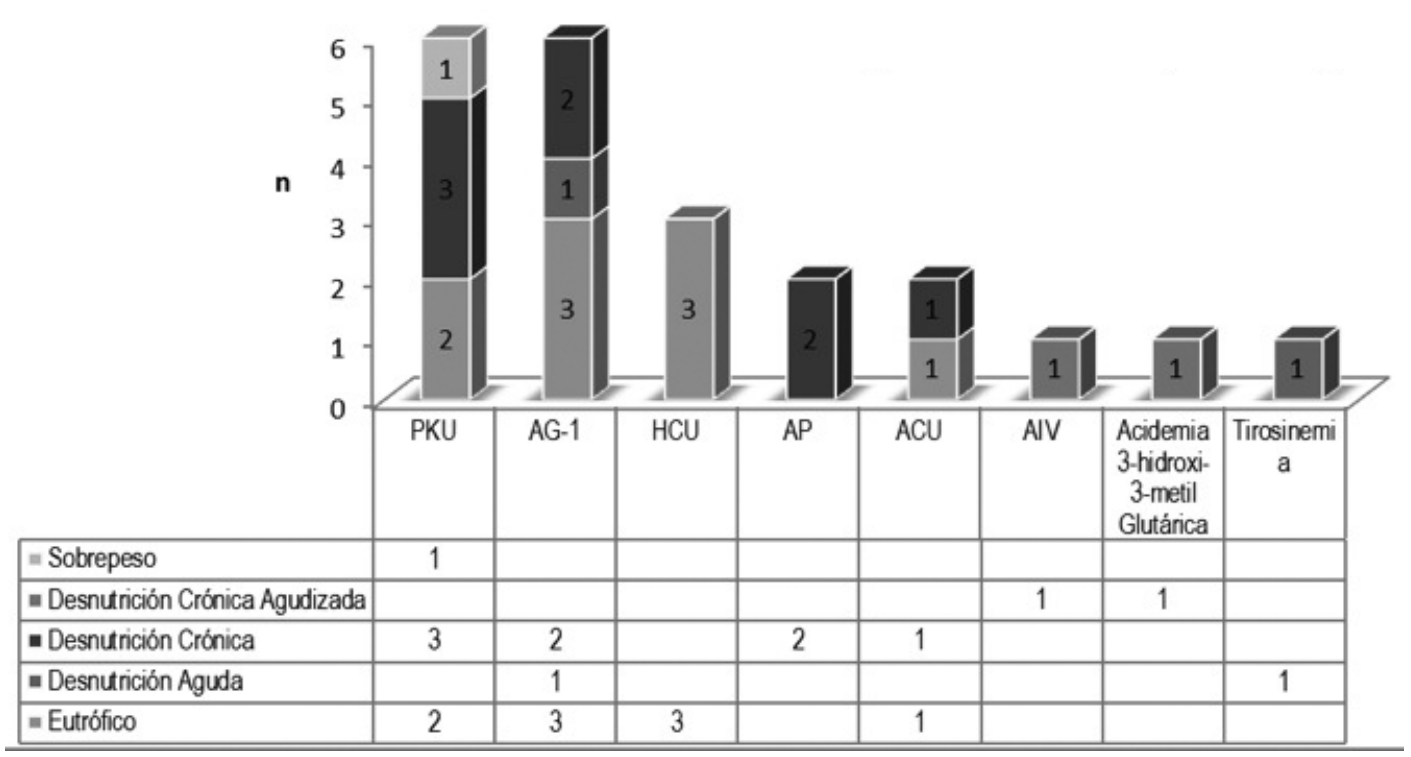


Al integrar los indicadores (gráfico 3) se detectó que 9 individuos (40\%) cursan con estado eutrófico mientras que para 10 de ellos (45\%) su principal afección es la talla sin estar afectado el peso.

En el gráfico 4 se observa el estado nutricional de los pacientes de acuerdo a su patología. Tanto en PKU, AG1 y $\mathrm{HCU}$ encontramos que predominantemente cursan con un adecuado estado nutricional. Sin embargo, el resto de las patologías presentan algún tipo de desnutrición, nuevamente siendo la talla la más afectada.

Al analizar el estado nutricional por grupos no se encontró diferencia por sexo, grupo etáreo y nivel socioeconómico; únicamente se encontró mayor proporción de individuos eutróficos en la zona rural (tabla 3 ).

Todos los pacientes tenían la prescripción dietaría según Nutrition Support Protocols Ross $® 10$, sin embargo a 3 pacientes (14\%) se les realizó la prescripción dietaria acorde a la edad correspondiente de su peso. De los 22 pacientes, 20 (90\%) presentaron adherencia al plan de alimentación (identificada como 5 de 7 días a la semana por recordatorio de 24 horas), los otros 2 pacientes presentaron una pobre adherencia al plan de alimentación (identificada como $<3$ de 7 días a la semana por recordatorio de 24 horas), uno (5\%) secundario a un episodio infeccioso y otro (5\%) porque no tuvo acceso a

TABLA 3

Comparación del estado nutricional por variables categóricas

\begin{tabular}{|c|c|c|c|c|c|c|c|c|}
\hline \multirow{2}{*}{\multicolumn{3}{|c|}{ Variable }} & $\mathrm{n}$ & \multicolumn{5}{|c|}{ Diagnóstico nutricional } \\
\hline & & & & Eutrófico & $\begin{array}{c}\text { Desnutrición } \\
\text { aguda }\end{array}$ & $\begin{array}{l}\text { Desnutrición } \\
\text { crónica }\end{array}$ & $\begin{array}{l}\text { Desnutrición } \\
\text { crónica agudizada }\end{array}$ & Sobrepeso \\
\hline \multirow[t]{3}{*}{ Sexo } & \multicolumn{2}{|c|}{ Masculino } & 12 & 5 & 1 & 4 & 1 & 1 \\
\hline & \multicolumn{2}{|c|}{ Femenino } & 10 & 4 & 1 & 4 & 1 & 0 \\
\hline & \multicolumn{2}{|c|}{$p$-value } & & 0.937 & 0.892 & 0.746 & 0.892 & 0.350 \\
\hline \multirow[t]{3}{*}{ NSE } & \multicolumn{2}{|c|}{ Bajo } & 17 & 6 & 1 & 7 & 2 & 1 \\
\hline & \multicolumn{2}{|c|}{ Medio y alto } & 5 & 3 & 1 & 1 & 0 & 0 \\
\hline & \multicolumn{2}{|c|}{$p$-value } & & 0.323 & 0.361 & 0.387 & 0.421 & 0.579 \\
\hline \multirow[t]{3}{*}{ Vivienda } & \multicolumn{2}{|c|}{ Rural } & 9 & 6 & 1 & 5 & 1 & 0 \\
\hline & \multicolumn{2}{|c|}{ Urbana } & 13 & 3 & 1 & 3 & 1 & 1 \\
\hline & \multicolumn{2}{|c|}{$p$-value } & & 0.041 & 0.784 & 0.119 & 0.784 & 0.394 \\
\hline \multirow[t]{4}{*}{ Grupo Etáreo } & \multicolumn{2}{|c|}{ Lactantes } & 8 & 2 & 2 & 3 & 1 & 0 \\
\hline & \multicolumn{2}{|c|}{ Preescolares y escolares } & 7 & 2 & 0 & 3 & 1 & 1 \\
\hline & \multicolumn{2}{|c|}{ Adolescentes y adultos } & 7 & 5 & 0 & 2 & 0 & 0 \\
\hline & \multicolumn{2}{|c|}{$p$-value } & & 0.137 & 0.146 & 0.854 & 0.594 & 0.325 \\
\hline \multicolumn{9}{|c|}{$\begin{array}{l}\text { Prueba Chi² } \\
\text { NSE: Nivel socioeconómico }\end{array}$} \\
\hline \multicolumn{9}{|c|}{ TABLA 4} \\
\hline \multicolumn{9}{|c|}{ Aporte energético y proteico } \\
\hline \multirow{2}{*}{\multicolumn{4}{|c|}{ Variables categóricas }} & \multicolumn{5}{|c|}{ Aporte dietético } \\
\hline & & & & $\begin{array}{r}\mathrm{Er} \\
(\mathrm{kcal}\end{array}$ & $\begin{array}{l}\text { ergía } \\
\text { kg/día) }\end{array}$ & p-value & $\begin{array}{l}\text { Proteína } \\
(\mathrm{g} / \mathrm{kg} / \mathrm{d})\end{array}$ & $p$-value \\
\hline \multirow{3}{*}{\multicolumn{2}{|c|}{ Sexo }} & Masculino & & 83. & $(92.7)$ & 0.6924 & $1.85(1.65)$ & 0.4674 \\
\hline & & Femenino & & 107 & (75.1) & & $3.2(2.1)$ & \\
\hline & & Total & & 102 & $(82.8)$ & & $2.8(1.9)$ & \\
\hline \multirow[t]{3}{*}{ NSE } & & Bajo & & 97. & $(68.1)$ & 0.9064 & $2.7(1.5)$ & 0.7835 \\
\hline & & Medio y alto & & 106. & (109.3) & & $3.2(2.1)$ & \\
\hline & & Total & & 102. & $5(82.8)$ & & $2.8(1.9)$ & \\
\hline \multirow{3}{*}{\multicolumn{2}{|c|}{ Vivienda }} & Rural & & 97.8 & $(58.3)$ & 0.6642 & $2.7(1.5)$ & 0.9733 \\
\hline & & Urbana & & 106 & (88.3) & & $3(2.1)$ & \\
\hline & & Total & & 102. & $5(82.8)$ & & $2.8(1.9)$ & \\
\hline \multirow{4}{*}{\multicolumn{2}{|c|}{ Grupo Etáreo }} & Lactantes & & 144 & $3(38.7)$ & 0.001 & $3.7(1)$ & 0.0038 \\
\hline & & Preescolares y es & colares & 97. & (31.9) & & $2.7(2.3)$ & \\
\hline & & Adolescentes y a & dultos & 38. & $(21.6)$ & & $1.7(0.4)$ & \\
\hline & & Total & & 102 & $(82.8)$ & & $2.8(1.9)$ & \\
\hline
\end{tabular}

Prueba de Mann-Whitney para comparer dos medianas (sexo, NSE y vivienda) y Prueba de Kruskal Wallis (grupo etáreo)

NSE: Nivel socioeconómico 
la formula especial. La adecuación no pudo ser determinada por patrones bioquímicos dado que no están disponibles en Colombia o no se tuvieron con la prontitud necesaria.

La ingesta energética promedio fue de $1570 \pm 547.2 \mathrm{Kcal} /$ día (rango entre 681 y 2758 kcal) o 95.6ะ49.6Kcal/Kg/día (rango entre 27 y 190), correspondiendo a lo recomendado según el promedio y rango de edad. La dieta de los pacientes presentó una distribución del valor energético total en promedio del 12\% de proteínas (3 g/Kg/día y 52 g/día), 34\% de lípidos (4 g/Kg/día y 57 g/día), y 54\% (13 g/Kg/día y 212 g/día) de hidratos de carbono. En cuanto al aporte de proteínas dadas por la fórmula especial libre de aminoácidos correspondientes según EIM y el aporte de proteínas dadas por la fórmula láctea infantil si correspondía, se encontró que en promedio los pacientes reciben $67 \%$ y $9 \%$ de la proteína respectivamente, y el resto de la proteína aportada por alimentos.

Al analizar el aporte energético de la dieta se encontró que era ligeramente mayor en mujeres que en hombres, en nivel socioeconómico medio y alto así como en aquellos con vivienda urbana; sin embargo, ninguna de estas diferencias fueron significativas. Por otro lado, el análisis por grupo etáreo demostró que, como era esperado, el aporte disminuye con la edad ya que éste es dependiente del peso (tabla 4). No fue posible realizar la comparación de aporte dietético por patología ya que en nuestra muestra tres patologías tenían menos de un individuo.

Para el aporte de aminoácidos dependiendo del EIM, se encontró que los pacientes cumplían con la cantidad recomendada de aminoácidos limitantes según Nutrition Support Protocols Ross $®(16)$. Asimismo, en cuanto a la suplementación de algunos cofactores los pacientes con Homocistinuria tenían indicado: piridoxina $25-1000 \mathrm{mg} /$ día, riboflavina $100-300 \mathrm{mg} /$ día para aquellos con AG1 y biotina $5-10 \mathrm{mg} /$ día para $A P$; sin embargo, pacientes con PKU no recibían tirosina.

\section{DISCUSIÓN}

Patologías crónicas e innatas como los EIM no sólo son causantes de daño neurológico sino que también se asocian a deficiencias nutricionales y sus consecuencias en el estado nutricional. El oportuno diagnóstico, tratamiento así como la adherencia a éste son esenciales para prevenir las secuelas neurológicas y alteraciones del estado nutricional, asegurando un crecimiento y desarrollo adecuado.

En los niños estudiados se encontró que cerca del $40 \%$ $(n=9)$ cursaba con estado nutricional eutrófico. La principal afección nutricional encontrada fue la talla, siendo que para pacientes pediátricos $(n=15)$ el z-score de T/E $(-1.9 \pm 1.6)$, indica riesgo de talla baja, razón por la cual 9 de 22 individuos cursaban con algún tipo de desnutrición crónica. La evaluación de peso por indicadores $\mathrm{P} / \mathrm{E}, \mathrm{P} / \mathrm{T}, \mathrm{IMC} / \mathrm{E}$ e IMC concluyó que 4 de los pacientes (18\%) sufría algún tipo de desnutrición aguda y que $1(4.5 \%)$ tuvo sobrepeso.

La alta prevalencia de pacientes eutróficos puede atribuirse a que se atendían en una consulta de nutrición especializada, con acceso a un mejor tratamiento. Es necesario considerar el error que implica incluir pacientes adultos (dos con PKU y dos con homocistinuria), en los cuáles no se evaluaba la talla rutinariamente como evaluación del estado nutricional. La mayoría (90\%) de los pacientes fueron de diagnóstico tardío (más de dos años de edad) por lo que la afección de crecimiento pudo existir desde temprana edad. El 10\% de los pacientes con diagnóstico neonatal fueron los de AG1.

En individuos con PKU se ha reportado retraso en el crecimiento durante la edad pediátrica, incluso en pacientes con diagnóstico oportuno y apego al tratamiento (11). Dicho retraso del crecimiento no se ha asociado a la concentración de fenilalanina por lo que se ha sugerido que la suplementación de ciertos micronutrimentos (como el zinc) así como del aminoácido limitante (tirosina) puede ser benéfico. En nuestra muestra, ningún paciente con PKU fue suplementado con tirosina ni ningún otro micronutrimento por lo que es posible sospechar que los pacientes adultos cursaron con talla baja durante la edad pediátrica.

Es necesario evaluar los datos en edad pediátrica para conocer el estado nutricional durante dicha etapa para poder concluir. Sin embargo al no contar con dichos datos debemos evaluar si la talla actual es considerada baja o no. En países como México se considera talla baja en el adulto cuándo ésta se encuentra por debajo de $1.50 \mathrm{~m}$ en mujeres y $1.60 \mathrm{~m}$ en hombres (12) por lo que uno de los individuos con PKU si presentaba talla baja (varón de $1.55 \mathrm{~m}$ ) mientras que el otro se encontraba limítrofe (femenino $1.52 \mathrm{~m}$ ).

En individuos con homocistinuria no se ha reportado afección de la talla, siendo confirmado en nuestra muestra donde incluso el paciente en edad pediátrica se encuentra eutrófico. Los dos individuos adultos con esta patología, a diferencia de los individuos PKU, no presentaban afección de talla ya que alcanzaron tallas de $1.75 \mathrm{~m}$ y $1.92 \mathrm{~m}$.

Otra debilidad de nuestro estudio al evaluar la talla fue que en tres pacientes (dos con acidemia glutárica y uno con tirosinemia) no se obtuvo dicha medición antropométrica. Las patologías de estos individuos se han asociado a mayor riesgo nutricional y alteraciones en crecimiento y desarrollo13 por lo que la evaluación nutricional tiene un alto riesgo de error al reportar la frecuencia con la que se presentó el estado eutrófico (pudiendo ser menor) o desnutrición crónica (pudiendo ser mucho mayor)

La alteración nutricional en patologías como las acidemias orgánicas y alteraciones del ciclo de la urea en las que sólo se presentó un paciente eutrófico nos llevan a pensar que son las patologías con mayor riesgo de muerte las que conducen a mayor riesgo nutricional. En el caso de las acidemias orgánicas, como la AP y AIV, se ha reportado retraso del crecimiento hasta en $40 \%$ de la población (14). Dicho estado nutricional se ha atribuido a la academia con la que cursan los pacientes, los recurrentes eventos agudos catabólicos y la acumulación de metabolitos secundarios responsables de anorexia y mala adherencia al tratamiento. Por dicha razón, estos pacientes usualmente requiere soporte nutricional enteral por gastrostomía y mejoran significativamente su estado nutricional (15).

De igual forma, las ACU suelen cursar con retraso en el crecimiento secundario a la restricción proteica así como los eventos catabólicos e hiperamonémicos que pueden sufrir (16).

El estado nutricional de nuestra muestra es independiente del sexo, nivel socioeconómico y grupo etáreo, Ilama la atención éste último ya que se esperaría que a mayor edad, mayor exposición a deficiencias nutricionales y mayor prevalencia de desnutrición. Encontramos que vivir en zona rural se asociaba a mayor proporción de pacientes eutróficos. Esta característica no necesariamente representa lo que ocurre en la generalidad de pacientes con EIM ya que la población rural de nuestra muestra vivía predominantemente en Bogotá.

En la dieta, el aporte energético fue de 102,2(82.8) kcal/ $\mathrm{kg} / \mathrm{d}$ y proteico de $2,8(1,9) \mathrm{g} / \mathrm{kg} / \mathrm{d}$. Dicho aporte cubría los requerimientos sugeridos por los protocolos de Nutrición de Ross ${ }^{\circledR}(16)$ de acuerdo a las categorías estáreas. No se encontraron diferencias entre sexos, NSE ni zona de vivienda. La disminución significativa del aporte energético y proteico por 
grupo etáreo era de esperarse dado que los requerimientos dependientes de peso disminuyen con la edad.

Dado el número limitado de la muestra y por patología, no fue posible analizar el aporte dietético por cada una de éstas ni por diagnóstico temprano ( 2 individuos) en comparación a tardío (20 indiviudos). Al agruparlas por EIM de proteínas que no producen hiperamonemia (PKU, tirosinemia y homocistinuria) y las que producen hiperamonemia ( $A C U$ y acidemias) no se encontraron diferencias en los aportes dietéticos.

Incrementar el tamaño muestral permitiría encontrar las diferencias por patologías así como su asociación con el estado nutricional ya que se esperaría encontrar en las ACU un aporte proteico significativamente menor.

En países como Colombia el diagnóstico oportuno se limita a la población privilegiada por lo que el diagnóstico usualmente es tardío y el tratamiento aún más. Asimismo, la disponibilidad de fórmulas especiales puede limitar la adherencia al tratamiento, favoreciendo las alteraciones nutricionales. En nuestros niños encontramos que el apego al tratamiento nutricional es alto ya que 19 de 22 se apegan a las recomendaciones nutricionales. Es importante destacar que únicamente un paciente tuvo dificultad por conseguir la fórmula especializada. Idealmente, la adhesión al tratamiento se evalúa con controles bioquímicos los que no están disponibles en nuestro medio o no se obtienen con la prontitud necesaria.

La alta frecuencia con la que se presenta el estado nutricional eutrófico se puede explicar, en parte, gracias a los aportes dietéticos adecuados que cubren el requerimiento (energético, proteico y de aminoácidos limitantes) así como la disponibilidad de la fórmula y la adherencia al tratamiento.

Si lo mencionado anteriormente fuera la solución al estado nutricional de los pacientes con EIM, nuestra población entera cursaría con un estado nutricional óptimo. Sin embargo, no se cuenta con controles bioquímicos con la frecuencia necesaria para poder realizar los ajustes dietéticos pertinentes en relación al estado metabólico de los pacientes. Esta debilidad de nuestro medio puede ser la responsable de que el tratamiento no sea siempre exitoso y que aún se presenten alteraciones del estado nutricional tanto agudas como crónicas.

Si bien la muestra analizada no fue representativa de los EIM más prevalentes en Colombia pero es un acercamiento a este tipo de población de la que muy poco se sabe en países en los que el tamizaje neonatal no es obligatorio. Nuestro estudio se limita a describir la situación nutricional de pacientes que tienen diagnóstico y que son tratados en un centro médico especializado. Los resultados descritos son producto de una medición transversal por lo que es de suma importancia realizar un estudio longitudinal que demuestre que el tratamiento nutricional es pilar en el manejo de estas patologías. Asimismo, se requiere de un grupo control sano, idealmente pareado, para descartar que la situación aquí presentada sea un reflejo de la situación nacional.

\section{CONCLUSIONES}

La consulta en nutrición especializada permite un adecuado manejo y seguimiento nutricional. Si éste se acompaña de adherencia al plan nutricional y evaluación bioquímica permitiría obtener un óptimo estado nutricional.

Este estudio demostró que a pesar de las diferencias en variables como sexo, NSE, zona de vivienda y grupo etáreo, cerca de la mitad presenta afección de talla y el $40 \%$ se encuentra eutrófico. Estos extremos se atribuyen por el tipo de EIM con el que cursan así como el apoyo nutricional que reciben. Asimismo, creemos que la frecuencia con la que se presenta la talla baja puede ser mayor considerando las limitantes del estudio.

Es necesario, realizar un estudio longitudinal que permita elucidar la variabilidad en el estado nutricional de estos pacientes e integrarlo con controles bioquímicos que permitan correlacionar el control metabólico con el estado nutricional y la adherencia al tratamiento.

Estudios de este tipo permitirán proveer evidencia a los países sin programa de tamizaje neonatal sobre la relevancia de estas pruebas así como el pilar que es la nutrición en el manejo de estos pacientes.

\section{RESUMEN}

Introducción: Poco se sabe sobre las secuelas nutricionales de los errores innatos del metabolismo (EIM) sin diagnóstico oportuno en Colombia. Objetivo: Describir las principales características nutricionales de pacientes con EIM de proteínas en consulta privada de nutrición en Bogotá, Colombia y si se atribuyen a la patología o variables sociodemográficas. Metodología: Se realizó un estudio descriptivo transversal observacional de pacientes con EIM de proteínas, recolectando datos sociodemográficos, antropométricos y dietéticos. Resultados: Se recolectaron 22 pacientes: 45\% de sexo femenino, 36\% lactantes; $59 \%$ de zona urbana y $77 \%$ de nivel socioeconómico (NSE) bajo. El 41\% se encontraba eutrófico y $45 \%$ presentó retraso de talla, sin existir diferencias por sexo, NSE, zona de residencia ni grupo etáreo. El 90\% presentó adherencia al tratamiento, cubriendo las recomendaciones para edad, patología y aminoácidos limitantes. No existieron diferencias significativas en el aporte dietético por variables sociodemográficas excepto por grupo etáreo. Conclusión: La principal afección nutricional es crónica y atribuible al EIM.

Palabras clave: errores innatos del metabolismo; antropometría, evaluación dietética, talla baja: tratamiento nutricional, manejo dietoterapéutico.

\section{BIBLIOGRAFÍA}

1. Ruiz M, Sánchez F, Dalmau J, Gómez L. Tratamiento nutricional de los errores innatos del metabolismo. 2da edición. Madrid, España: 2007:4-32.

2. Colombo M, Cornejo V, Rainmann E, editoras. Errores innatos en el metabolismo del niño. Segunda edición. Editorial universitaria: Santiago de Chile 2010: 47-69.

3. Acosta P. Yannicelli S. Nutrition Support Protocols, 4th edit., Ross Products Division, Columbus, Ohio, USA, 2001.

4. Ruiz M, Santana C, Trujillo R, Sánchez F, Dalmau J. Aproximación al tratamiento nutricional de los errores innatos del metabolismo (III). Acta pediátrica española. 2002; 60: 34-40.

5. Gomez F. Desnutrición. Boletín Medico Hospital Infantil de México 1991; 54: 299-304.

6. Waterlow. Clasification and definition of protein-calorie malnutrition. British Med J 1972; 3: 566-9.

7. Onis M, Onyango A, Borghi $E$, et al. Development of a WHO growth reference for school-aged children and adolescents. Bulletin of the WHO 2007; 85 (9):660-7.

8. Consenso de la Sociedad Española para el Estudio de la Obesidad SEEDO' 2000 para la evaluación del sobrepeso y la obesidad y el establecimiento de criterios de intervención terapéutica. Med Clin (Barc) 2000; 115: 587-597.

9. Frisancho AR. Triceps skin Fol. And upper arm muscle size normal for assessment of nutricional status. Am J Clin Nutr 1974, 27:1052-8. 
10. Acosta PB, Yannicelli S. The Ross Metabolic Formula System Nutrition Support Protocols. Abbott Laboratories, 4th Edition. 2001.

11. Dobbelaere D, Michaud L, Debrabander A, et al. Evaluation of nutritional status and pathophysiology of growth retardation in patients with phenylketonuria. J Inherit Metab Dis 2003;26(1):1-11.

12. Norma Oficial Mexicana de Sobrepeso y Obesidad. NOM174-SSA1-1998. Secretaria de Salud, 1998. Disponible en: http://www.salud.gob.mx/unidades/cdi/nom/174ssa18. htm
13. Yannicelli S, Rohr F, Warman ML. Nutrition support for g/utaric academia type I. J Am Diet Assoc 1994; 94(2):183-8.

14. de Baulny HO, Benoist JF, Rigal O, et al. Methylmalonic and propionic acidaemias: management and outcome. J Inherit Metab Dis 2005; 28(3): 415-23.

15. Cornejo V, Colombo M, Duran G, et al. Diagnosis and follow up of 23 children with organic acidurias. Rev Med Chil 2002; 130(3): 259-66.

16. Bachmann C. Outcome and survival of 88 patients with urea cycle disorders: a retrospective evaluation. Eur J Pediatr 2003; 162:410-6. 